\title{
Lighting Consumption Optimization in a SCADA Model of Office Building Considering User Comfort Level
}

\author{
Mahsa Khorram, Pedro Faria, Zita Vale \\ GECAD - Research Group on Intelligent Engineering and Computing for Advanced Innovation \\ and Development, Institute of Engineering, Polytechnic of Porto (ISEP/IPP), Portugal \\ \{makgh, pnfar, zav\}@isep.ipp.pt
}

\begin{abstract}
Due to the high penetration of the buildings in energy consumption, the use of optimization algorithms plays a key role. Therefore, all the producers and prosumers should be equipped with the automation infrastructures as well as intelligent decision algorithms, in order to perform the management programs, like demand response. This paper proposes a multi-period optimization algorithm implemented in a multi-agent Supervisory Control and Data Acquisition system of an office building. The algorithm optimizes the lighting power consumption of the building considering the user comfort constraints. A case study is implemented in order to validate and survey the performance of the implemented optimization algorithm using real consumption data of the building. The outcomes of the case study show the great impact of the user comfort constraints in the optimization level by respect to the office user's preferences.
\end{abstract}

Keywords: multi-period optimization, SCADA, user comfort, office building

\section{Introduction}

Every day, a lot of energy is lost by the negligence of people around the world. Sometimes the unimportant actions during a day can be the terminator of the environment and earth at the end [1]. That is why the world is moving towards comprehensive automation and smart infrastructure in the buildings, in order to prevent the loss of energy as much as possible [2]. In addition to these facilities, Demand Response (DR) programs organize the user's consumption pattern as a generic and systematic program according to electricity price variations or technical issues with considering consumers and producers interests. DR programs have a desirable variety which is divided into two main groups, namely price-based demand response and incentive-based demand response [3].

The buildings are responsible for $40 \%$ of world energy consumption which is increasing every day [4]. Among all types of buildings, office buildings can be considered

The present work was done and funded in the scope of the following projects: COLORS Project PTDC/EEI-EEE/28967/2017 and UID/EEA/00760/2019 funded by FEDER Funds through COMPETE program and by National Funds through FCT. 
as a more flexible option for implementing DR programs, since usually they have significant energy consumption, and also in some cases can be more equipped to automation infrastructure than residential houses.

Recently, the main concern in energy minimization topics is respect to user comfort while energy consumption is optimizing [5]. keeping a balance between energy minimization and user preferences need a formulation with precise restrictions in order to observe optimization purposes and user easement at the same time [6].

Mostly in office buildings, more attention is paid to Air Conditioners (AC) while $29 \%$ of total energy consumption in office buildings belongs to the lighting system [7]. The lights of an office building can be considered as flexible loads for reduction and curtailment if they are fully controllable and reducible by existing equipment. Supervisory Control And Data Acquisition (SCADA) system play a key role in DR implementation since it offers various advantages in order to have automatic load control in different types of buildings [8]. For instance, the SCADA system can dominate the lights of the illumination system which they are fully controllable via the Digital Addressable Lighting Interface (DALI) [8].

In this matter, SCADA systems can be integrated with the Multi-Agent Systems (MAS) for improving the overall system performance. If the SCADA system is equipped with the MAS, various types of optimization algorithms could be solved and utilized by the model in order to control and manage the resources controlled by the SCADA system [9]. Agent-based SCADA models proyide more flexibility and adaptability [10].

This paper proposes a multi-period optimization algorithm for the lighting system of an office. The algorithm focuses on the minimization of the power consumption of the lights with respect to user comfort. The algorithm is implemented in an agent-based SCADA system installed in the office building. All the parameters in the building, such as the consumption of each light and total consumption as well, are monitored through this SCADA system.

Several studies have been done in the context of building energy optimization. In [7], the authors proposed a smart lighting control based on internal mode controller of an artificial neural network which tries to maintain occupant's preferences while are using natural light at the same time. In [8], presented a SCADA-based model focused on the lights and ACs consumption of the building for participating in DR events. In [11], ACs and lights consumption is managed and minimized under Real Time Pricing (RTP) tariffs in a MAS based SCADA model. A lighting consumption optimization has been proposed in [12] by considering the renewable resources. In [13], the user satisfaction provided based on time and device, while user budget is considered. However, the focus of this paper is to study the impact of the user comfort constraints considered for the proposed optimization algorithm. The outcomes of optimization would be compared and surveyed with and without considering the user comfort constraints.

After this section, the optimization algorithm and the implemented methodology is explained in Section 2. A case study is demonstrated in Section 3 in order to validate the performance of the proposed optimization algorithm, and the gained results will be compared in the same section. Finally, Section 4 describes the main conclusions of the work. 


\section{Optimization Algorithm}

This section presents the optimization algorithm implemented in the SCADA system. As it was described, the SCADA model is agent-based, with a various number of agents and players. The system was developed by the authors in the scope of their previous works [11], and this section focuses only on the Optimizer agent and the implemented optimization algorithm on this agent. More details and information regarding the SCADA model and the other agents are available on [11].

The main purpose of the algorithm is minimizing the power consumption of the lights with respect to user comfort. Each light participates in minimization call from optimizer agent as a component of the system. For each light, an importance weight is dedicated by numbers between 0 and 1 , in order to define the priority of them. These numerical criteria are dependence to several conditions such as preferences of the user, the location of the light and operation of natural daylight. These priority numbers observe the user comfort to some extent but, more restrictions are required to prevent any exorbitance reduction. For this purpose, several constraints are provided to limit power reduction more than enough. Since the present algorithm is a multi-period optimization algorithm, there is full control on each light in all periods. Therefore, the algorithm can prevent reduction more than enough from only some particular lights in continues periods. It means the situation of each light changes during all periods by comfort constraints and priority numbers.

Fig. 1 illustrates the algorithm of the present methodology with detailed steps. It should be noted that to achieve the algorithm purposes, the cooperation of all components of the agents are required for providing essential input data. Initial data such as rated power consumption of the lights, nominal power consumption of the lights, and power consumption of other existing devices in the building are the pre-optimization requirements that should be provided by SCADA system and the other agents.

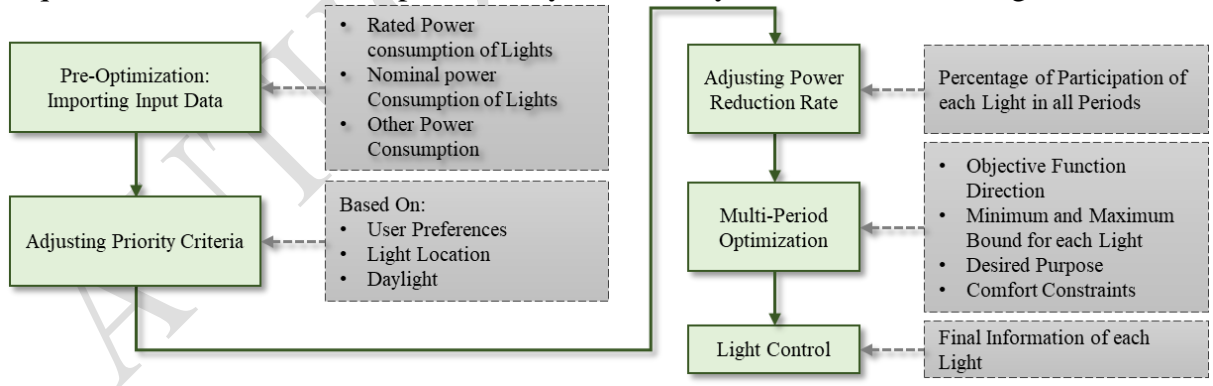

Fig. 1. The procedure of optimization algorithm.

Priority numbers are determinative parameters in optimization algorithm which can set the role of each light in the optimization. As can be seen in Fig. 1, these priority numbers are subject to several issues such as user preferences, light location, and natural light. Another parameter that makes the reduction in lights more rigid is the power reduction rate for each light in all periods. This parameter limits the power reduction in each particular light in all periods and causes the reduction to be divided into all of the 
lights. After defining these parameters, variables should be bounded and the relative constraints should be defined. The desired purpose of this optimization algorithm is reducing specified power reduction with observing all the existing constraints. This desired power reduction can be determined according to several aspects such as electricity price variation, ON-Peak or OFF-Peak hours, rated power generation, and energy storages if exist. After specifying all the required data, the algorithm runs, and the results are visible.

The proposed methodology is defined as a Linear Programming (LP) optimization problem, which is modeled via "OMPR" package of Rstudio ${ }^{\circledR}$ (www.rstudio.com) and is solved via "GLPK" library.

The Objective Function (OF) of the proposed optimization algorithm is shown in (1) in order to minimize the power consumption of the lights.

$$
\text { Minimize OF }=\sum_{t=1}^{T} \sum_{l=1}^{L} \text { Priority }_{(l, t)} \times P_{(l, t)}
$$

Priority is the number between 0 and 1 that is dedicated to each light for representing the importance of each light for the users and the bigger priority numbers are allocated to more important lights. $P$ is the decision variable of the algorithm that shows the amount of power that should be reduced from each light in each period. It should be noted that $T$ and $L$ are the maximum number of periods and lights respectively.

The definition of upper bounds related to the amount of power reduction and priority of each light in each period are developed in the scope of the author's previous work [12], and they are not mentioned in this section.

Equation (2) is modeled to show the total power reduction in all the lights in each period. The objective function in (1) is subject to (2) and (3).

$$
\sum_{l=1}^{L} P_{(l, t)}=R R_{(t)}
$$

$R R$ is an abbreviation of Required Reduction in each period from all the lights. Equation (3) makes each light restricted individually by the power reduction rate coefficient in order to maintain user comfort. It means the total power reduction of each light in all periods can be adjusted and limited by (3).

$$
\begin{aligned}
\sum_{t=1}^{T} P_{(l, t)} & =P R R_{(l)} \times \sum_{t=1}^{T} \text { init. } P_{(l, t)} \\
& \forall l \in\{1, \ldots, L\}
\end{aligned}
$$

$P R R$ is brevity of Power Reduction Rate for light. $P R R$ can be as a percentage of total actual power consumption of each light in all periods. The initial power consumption of each light is shown by init.P that means the power consumption of light in a normal situation and without any reduction. Also, as it was shown on (3), the power reduction of each light in all periods cannot exceed a defined limitation for observing user comfort. PRR can make power reduction rigid in several ways. It can be used as a coefficient for each light individually. It also can be also as a function of time. For instance, it can limit the power reduction of certain lights in certain periods of time. 


\section{Case Study and Results}

An office building is considered that its illumination system based on fluorescent lights with DALI ballasts. The building includes 8 offices and one corridor. Each office has two 100W lights, and the corridor contains four $100 \mathrm{~W}$ lights. The present study considers 20 controllable and reducible lights. Fig. 2 illustrates the plan of the building.

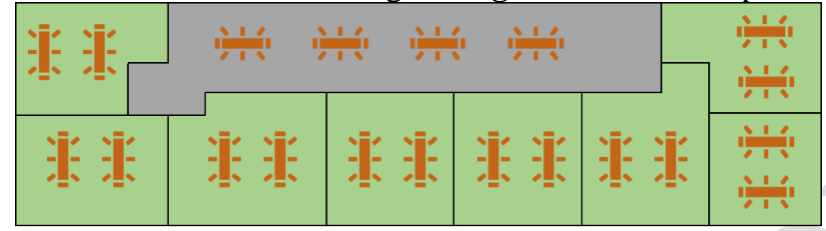

Fig. 2. Plan of the office building with 20 lights.

The total consumption of the lighting system in the building will be $2000 \mathrm{~W}$. However, the minimum reduction for each light is supposed to equal to zero, while the maximum reduction stays for $65 \%$ of nominal consumption of the light. It should be noted that, in order to avoid turning off any light completely, the maximum reduction is bounded. The algorithm surveys from 8 am to $8 \mathrm{pm}$ with 15 minutes time intervals. According to the day-ahead data, the initial consumption of the lights and non-controllable consumption of the building is shown in Fig. 3 for 48 periods. The Required Reduction (RR), can be determined based on diverse issues such as difference in production and generation rate in the building, or existing power in energy storages.

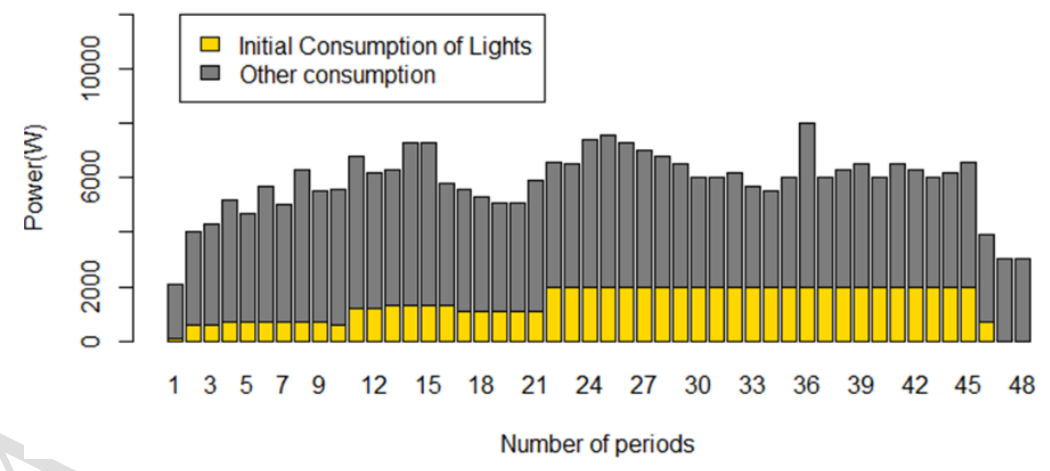

Fig. 3. The classified initial consumption of the building.

According to Fig. 3, the power consumption of the lights has been varied during the day and in periods 47 and 48 all the lights have been turned off. Since the Power Reduction Rate (PRR) is an essential parameter that impresses user comfort directly, in each execution, different values of PRR are considered in order to validate the impact of comfort constraints more precisely. The result of the first execution are in Fig. 4 while the constraint demonstrated by (3) is ignored. As seen in Fig. 4 - (A), the power reduction in some lights, such as $\mathrm{L} 7, \mathrm{~L} 9$, and L12, is much more than the other ones. Therefore, Fig. 5 shows the output of the next implementation of algorithm while PRR defined to $35 \%$ for all the lights. 

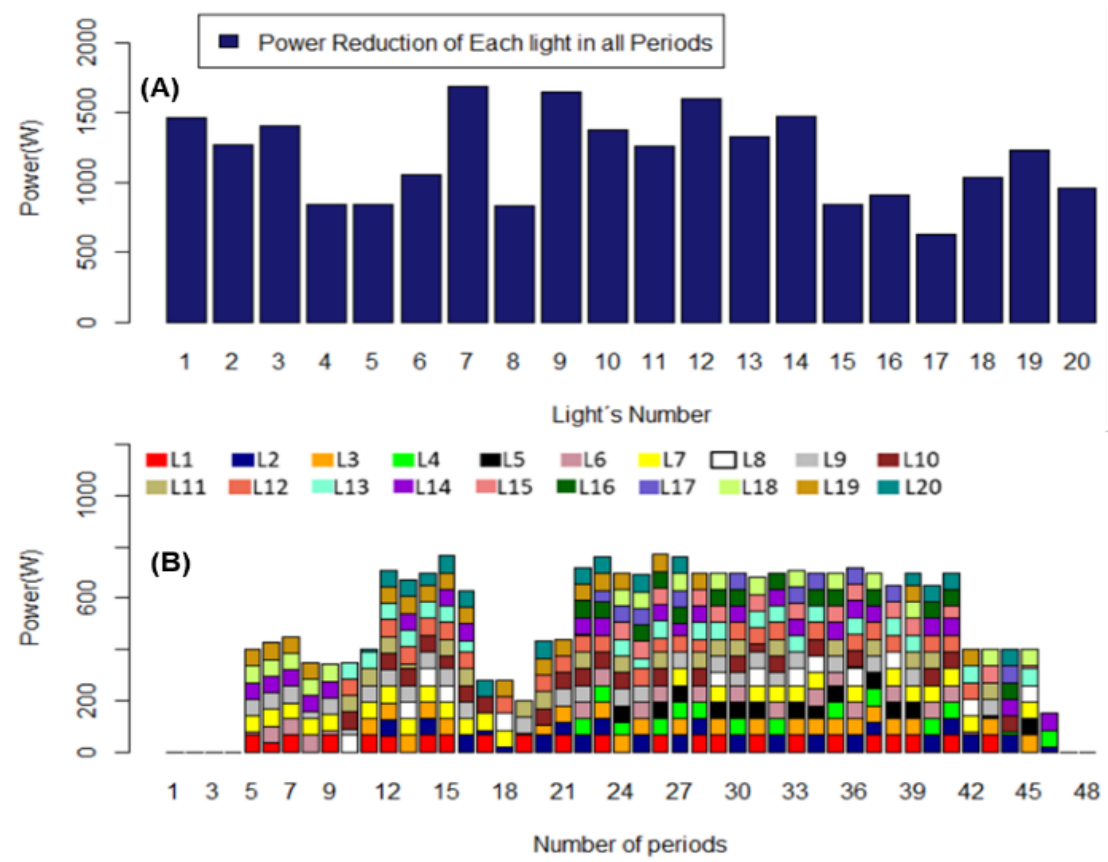

Fig. 4. Optimization results without considering user comfort constraint; (A) sum of power reduction of each light in all periods, (B) power reduction from all lights in each period.
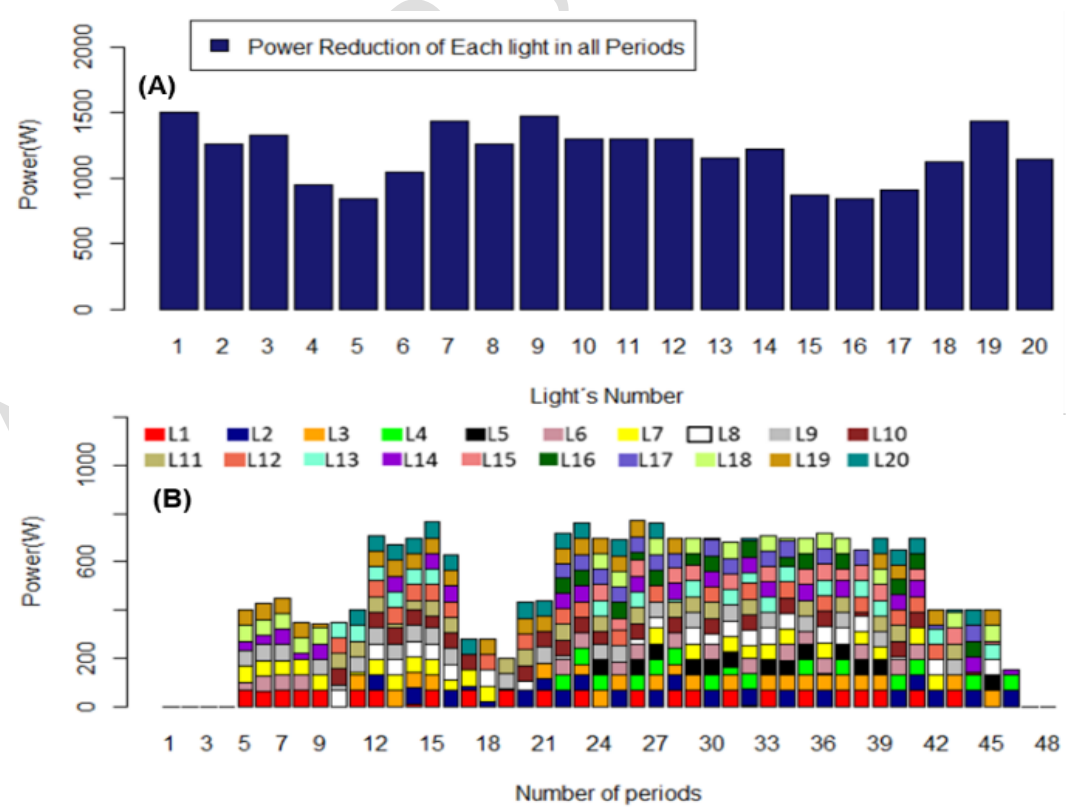

Fig. 5. Optimization results with $35 \%$ user comfort level; (A) sum of power reduction of each light in all periods, (B) power reduction from all lights in each period. 

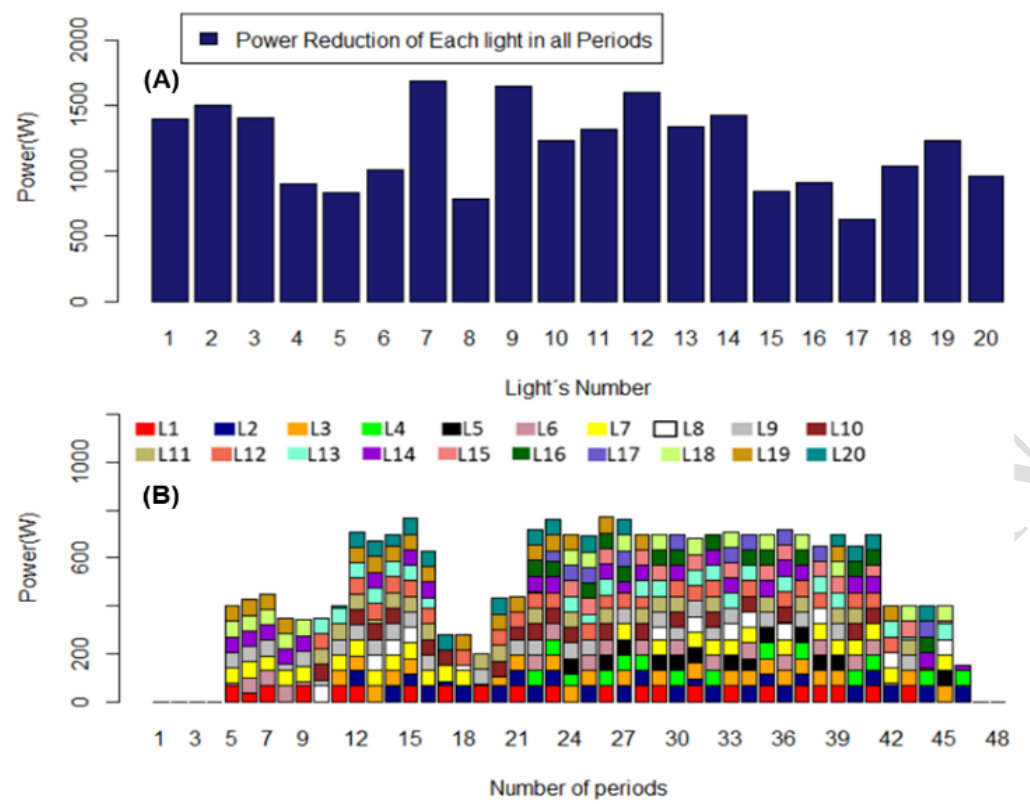

Fig. 6. Optimization results with $50 \%$ user comfort level; (A) sum of power reduction of each light in all periods, (B) power reduction from all lights in each period.

According to Fig. 6, power reduction in each light has been slightly changed when PRR is equal to $50 \%$. By comparing the results in this section, the importance of the parameter PRR is obvious. In this case, while the PRR is on $35 \%$, the optimization algorithm has the best performance, somehow, it reduced the amount of power reduction by maintaining a high level of user comfort. The balanced cooperation of the lights is obviously visible in Fig. 5 while the required reduction is constant. Fig. 6 presents obtained results when PRR is equal to $50 \%$.

\section{Conclusions}

Due to the high penetration of the buildings in energy consumption, the use of optimization algorithms plays a key role. Therefore, all the producers and prosumers should be equipped with the automation infrastructures as well as intelligent decision algorithms, in order to perform the management programs, like demand response.

A multi-period optimization algorithm has been proposed in this paper to minimize the energy consumption of the lights with considering user comforts. In addition to the definition of lights priorities, a power reduction rate parameter has been defined with a direct impact on user comfort. The algorithm was implemented in a multi-agent Supervisory Control and Data Acquisition system of an office building. The focus of this paper was given to the Optimizer agent, where the developed algorithm was executed in order to optimize the consumption of the lighting system in the building. 
In the case study of this work, the impact of the developed optimization algorithm is demonstrated during the working hours. A key parameter in the user comfort constraint was changed in three levels, in order to validate the performance and select the most appropriate value for this case. The obtained results of the algorithm demonstrated and proved that how the used constraints made a balance on power reduction and user comfort level.

\section{References}

1. Chen, Y., Xu, P., Gu, J., Schmidt, F., Li, W.: Measures to improve energy demand flexibility in buildings for demand response (DR): A review. Energy and Buildings 177, 125-139, (2018).

2. Abrishambaf, O., Faria, P., Vale, Z.: SCADA Office Building Implementation in the Context of an Aggregator. IN: IEEE 16th International Conference on Industrial Informatics (INDIN), Porto, Portugal (2018).

3. Faria, P., Vale, Z.: Demand response in electrical energy supply: An optimal real time pricing approach. Energy 36(8), 5374-5384 (2011).

4. Cao, Y., Du, J., Soleymanzadeh, E.: Model predictive control of commercial buildings in demand response programs in the presence of thermal storage. Journal of Cleaner Production 218, 315-327 (2019).

5. Althaher, S., Mancarella, P., Mutale, J.: Automated Demand Response From Home Energy Management System Under Dynamic Pricing and Power and Comfort Constraints. IEEE Transactions on Smart Grid 6(4), 1874-1883 (2015).

6. Nguyen, D., Le, L.: Joint Optimization of Electric Vehicle and Home Energy Scheduling Considering User Comfort Preference. IEEE Transactions on Smart Grid 5(1) 188-199 (2014).

7. Kandasamy, N., Karunagaran, G., Spanos, C., Tseng, K., Soong, B.: Smart lighting system using ANN-IMC for personalized lighting control and daylight harvesting. Building and Environment 139, 170-180 (2018).

8. Khorram, M., Abrishambaf, O., Faria, P., Vale, Z.: Office building participation in demand response programs supported by intelligent lighting management. Energy Informatics 1(1), (2018).

9. Santos, G., Femandes, F., Pinto, T., Silva, M., Abrishambaf, O., Morais, H., Vale, Z.: House management system with real and virtual resources: Energy efficiency in residential microgrid. In: Global Information Infrastructure and Networking Symposium (GIIS), pp. 1-6, Porto, Portugal (2016).

10. Gazafroudi, A., Pinto, T., Prieto-Castrillo, F., Corchado, J., Abrishambaf, O., Jozi, A., Vale, Z.: Energy flexibility assessment of a multi agent-based smart home energy system. In: IEEE 17th International Conference On Ubiquitous Wireless Broadband (ICUWB), pp. 1-7, Salamanca, Spain (2017).

11. Khorram, M., Faria, P., Abrishambaf, O., Vale, Z.: Demand Response Implementation in an Optimization Based SCADA Model Under Real-Time Pricing Schemes. In: Advances in Intelligent Systems and Computing, 21-29 (2019).

12. Khorram, M., Faria, P., Abrishambaf, O., Vale, Z.: Lighting Consumption Optimization in an Office Building for Demand Response Participation. In: Power Systems Conference Clemson University (PSC), Charleston, SC, USA (2018). 
13. Ogunjuyigbe, A., Ayodele, T., Akinola, O.: User satisfaction-induced demand side load management in residential buildings with user budget constraint. Applied Energy 187, 352366 (2017). 